Journal of Applied Sciences and Nanotechnology

Journal homepage: https://jasn.uotechnology.edu.iq/

\title{
A Generalization of the Concave Integral in Terms of Decomposition of the Integrated Function for Bipolar Scales
}

\author{
Fadheela Kareem*, Jabbar Abbas* \\ Department of Applied Sciences, University of Technology - Iraq
}

\section{Article information}

\section{Article history:}

Received: July, 07, 2021

Accepted: November, 16, 2021

Available online: December, 04, 2021

\section{Keywords:}

Capacities,

Concave integral,

Bi-capacities,

Bipolar concave integral,

Multiple-criteria decision analysis

*Corresponding Author:

Jabbar Abbas

jabbar.a.ghafil@uotechnology.edu.iq

\begin{abstract}
In the context of the multiple-criteria decision aid (MCDA), several fuzzy integrals concerning capacities (non-additive measures) have been introduced by various researchers in the last sixty years. Recently, Lehrer has proposed a new integral for capacities known as concave integral. The concave integral is based on the decomposition of random variables into simple ingredients. The concave integral concerning capacity is defined as the maximum value obtained among all its decompositions. The paper aims to model a new integration based on the decomposition of random variables into simple ingredients for multi-criteria decision making support when underlying scales are bipolar. This paper proposes a generalization of the concave integral in terms of decompositions of the integrated function to be suitable for bipolar scales. We show that the random variable is analyzed as a combination of indicators, where each allowed decomposition has a value determined by the bi-capacity. Lastly, we illustrate our framework by a practical example.
\end{abstract}




\section{Introduction}

The applications of fuzzy integrals are widely used in many fields, (e.g. image processing, face recognition, economic, computational intelligence, multi-criteria decision making problem, biology, education, finance, pattern recognition, data fusion, operations research, etc.). Several fuzzy integrals concerning capacities (non-additive measures) have been introduced by various researchers in the last sixty years [1-7]. The fuzzy integrals with respect to non-additive measures have been studied and applied in diverse fields [8-10].

Grabisch and Labreuche [11] proposed the concept of bi-capacity as a generalization of capacity. The bipolar fuzzy integrals for bi-capacities and their possible applications have been introduced and discussed in recent literature [12-14] as an extension of the fuzzy integral for cases in which the underlying scale is bipolar.

Recently, Lehrer [15] introduced a new integral for capacities based on a decomposition of random variables into simple ingredients. The concave integral for capacity is defined as the maximum value obtained among all its decompositions. In this paper, we propose a generalization of the concave integral in terms of decompositions of the integrated function to be suitable for bipolar scales. In this integration, we show that the random variable is analyzed as a combination of indicators, where each allowed decomposition has a value determined by the bi-capacity.

The paper is organized as follows. The following section recalls the background that is needed in this paper. Section 3 discusses the notion of bi-capacity. In section 4, we propose a generalization of the concave integral for bi-capacity. Section 5 gives a practical example of the generalization of the concave integral for bipolar scales. Section 6 presents the results and discussion of the application of the generalized concave integral. Lastly, the paper finished with some conclusions. Throughout the paper, $\mathbb{N}$ is the set of natural numbers, $R$ is the set of real numbers, $X=\left\{x_{1}, x_{2}, \ldots, x_{n}\right\}$ be a universal set of $n$ elements and $P(X)$ denotes the power set of $X$.

\section{Background and basic concepts}

In this section, we present the background of the study to the construction of the generalizations of the concave integral that we shall discuss in the paper.

Let $(X, \mathbb{S})$ be a measurable space, where $X$ is a non-empty set and $\mathbb{S}$ is a $\sigma$ - algebra

of subsets of $X$. A capacity [1] (or fuzzy measure [2]) is a generalization of classical measure using nonadditivity property instead of additivity property. The definition of the capacity is as follows.

Definition 1. [2] Let $X=\left\{x_{1}, x_{2}, \ldots, x_{n}\right\}$ be a universal set. A capacity is a function $\mu: \mathbb{S} \rightarrow[0,1]$ satisfies:

1. $\mu(\varnothing)=0, \mu(X)=1$,

2. for all $A, B \in \mathbb{S}, A \subseteq B, \mu(A) \leq \mu(B)$.

In decision analysis, especially in multiple-criteria decision analysis, has introduced several non-additive integrals for capacities in the last 60 years. One of them is concave integral known as Lehrer integral. The concave integral concept has been proposed in [15] as a novel approach to integrals for capacities, as follows.

Let $\mathbb{F}$ be a class of all finite nonnegative real-valued measurable functions on measurable space $(X, \mathbb{S})$. For any $f \in \mathbb{F}$, the sum $\sum_{\mathrm{i}=1}^{\mathrm{n}} \propto_{\mathrm{i}} \mathrm{I}_{\mathrm{A}_{\mathrm{i}}}$ is a decomposition of $f$, if $\propto_{\mathrm{i}} \geq 0$ for every $\mathrm{n} \in \mathbb{N}$ and 
$\sum_{\mathrm{i}=1}^{\mathrm{n}} \propto_{\mathrm{i}} \mathrm{I}_{\mathrm{A}_{\mathrm{i}}}=f$, where $I_{A_{i}}$ denoted to the indicator of $A_{i}$, which is the random variable that takes 1 over $A_{i}$, and value 0 otherwise. The concave integral of $f$ with respect to $\mu$ is an optimal decomposition $\sum_{\mathrm{i}=1}^{\mathrm{n}} \propto_{\mathrm{i}} \mu\left(\mathrm{A}_{\mathrm{i}}\right)$ among all decompositions of $f$, as given in the following definition.

Definition 2. [15] Let $(X, \mathbb{S})$ be a measurable space and $f \in \mathbb{F}$. The concave integral of $f$ respect to capacity $\mu$ is defined by

$$
\text { (L) } \int f d \mu=\bigvee\left\{\sum_{i=1}^{n} \propto_{i} \mu\left(A_{i}\right): \sum_{i=1}^{n} \propto_{i} I_{A_{i}} \leq f, \propto_{i} \geq 0, \quad\left\{A_{i}\right\}_{i=1}^{n} \subset \mathbb{S}, \quad n \in \mathbb{N}\right\} .
$$

\section{Definition of bi-capacities}

Even though capacities can catch a wide group of decision activities, they may be inefficient in some circumstances, specifically when the scales are bipolar. Hence, in many practical cases, it is natural to use a scale going from negative (bad) to positive (good) values, including a central neutral value, to encode the bipolarity of the effect. Such a scale is called a bipolar scale, typical examples are $[-1,1]$ (bounded cardinal), $R$ (unbounded cardinal) or \{very bad, bad, medium, good, excellent\} (ordinal). "Let us take for simplicity the $[-1,1]$ scale, with neutral value 0 ".

Then it is natural to use a scale going from negative (bad) to positive (good) values, including a central neutral value, to encode the bipolarity of the effect. Such a scale is called a bipolar scale, typical examples are $[-1,1]$ (bounded cardinal), $R$ (unbounded cardinal) or $\{$ very bad, bad, medium, good, excellent $\}$ (ordinal).

In a more general model, by considering that independence between positive and negative parts does not hold, so that we have to consider ternary alternatives $\left(1_{A},-1_{B}, 0_{(A \cup B}\right)^{c}$, and assign to each of them a number in $[-1,1]$. We denote this number as $\mu(A, B)$, i.e., a two-argument function, whose first argument is the set of totally satisfied criteria, and the second one the set of totally unsatisfied criteria, the remaining criteria being at the neutral level.

In the field of multi-criteria decision making, Grabisch and Labreuche[11] have presented the definition of bi-capacity as follows.

Let $Q(X)=3^{X}=\left\{\left(A_{1}, A_{2}\right) \in P(X) \times P(X) \mid A_{1} \cap A_{2}=\varnothing\right\}$ be the set all disjoint pairs of sets and equip it with a binary relation $\subseteq$ for arbitrary $\left(A_{1}, A_{2}\right),\left(B_{1}, B_{2}\right) \in Q(X)$ such that:

$$
\left(A_{1}, A_{2}\right) \sqsubseteq\left(B_{1}, B_{2}\right) \text { iff } A_{1} \subseteq B_{1} \text { and } A_{2} \supseteq B_{2}
$$

In this order on the structure $(Q(X), \sqsubseteq$ ) becomes a lattice. Sup and $\operatorname{Inf}$ are denoted by $\sqcup, \Pi$, respectively. The Supremum is given by

$$
\left(A_{1}, A_{2}\right) \sqcup\left(B_{1}, B_{2}\right)=\left(A_{1} \cup B_{1}, A_{2} \cap B_{2}\right)
$$


Infimum is given by

$$
\left(A_{1}, A_{2}\right) \sqcap\left(B_{1}, B_{2}\right)=\left(A_{1} \cap B_{1}, A_{2} \cup B_{2}\right)
$$

The top and bottom are, $(X, \varnothing) \&(\varnothing, X)$ respectively, (for more details [11]).

There is another order relation on the structure $Q(X)$ has introduced by

Bilbao et al. [12]. This order is simply the product order on $P(X) \times P(X)$ :

$$
\left(A_{1}, A_{2}\right) \subseteq\left(B_{1}, B_{2}\right) \text { iff } A_{1} \subseteq B_{1} \text { and } A_{2} \subseteq B_{2}
$$

Infimum is given by

$$
\left(A_{1}, A_{2}\right) \sqcap\left(B_{1}, B_{2}\right)=\left(A_{1} \cap B_{1}, A_{2} \cap B_{2}\right)
$$

always exists in $Q(X)$, while the Supremum does not exist in general. Hence, this ordered set is an infsemilattice with a bottom element $(\varnothing, \varnothing)$. For more details [16 \&17].

Definition 5. [4] A bi-capacity on $X$ is a set function $v_{b}: Q(X) \rightarrow[-1,1]$ if

$$
\begin{aligned}
& \text { 1- } v_{b}(\varnothing, \varnothing)=0, v_{b}(N, \varnothing)=1 \text { and } v_{b}(\varnothing, N)=-1 \\
& \text { 2- } \forall\left(A_{1}, A_{2}\right),\left(B_{1}, B_{2}\right) \in Q(X),\left(A_{1}, A_{2}\right) \sqsubseteq\left(B_{1}, B_{2}\right) \Longrightarrow v_{b}\left(A_{1}, A_{2}\right) \leq v_{b}\left(B_{1}, B_{2}\right) .
\end{aligned}
$$

\section{A generalization of concave integral}

In this section, we propose an expression of the bipolar concave integral in terms of decompositions of the integrated function as a generalization of the concave integral.

Let us denote by $I_{(A, B)}$ for the indicator of $(A, B)$, which is the random variable that takes 1 over $A,-1$ over $B$, and value 0 otherwise. That is,

$$
I_{(A, B)}= \begin{cases}1 & \text { iff } \quad i \in A \\ -1 & \text { iff } \quad i \in B \\ 0 & \text { otherwise }\end{cases}
$$

Where, $A$ represents the positive part and $B$ represent the negative part. Therefore, $\propto_{i} I_{\left(A_{i}, B_{i}\right)}$ is a decomposition of $f$. This means that a particular decomposition of $f$ is used to calculate the bipolar concave integral for the bi-capacity.

Thus, we can write the bipolar concave integral in terms of decompositions of the integrated function $f$ for $v_{b}$ as the following definition.

Definition 2. Let $v_{b}: Q(X) \rightarrow[-1,1]$ be a bi-capacity and $f \in \mathbb{F}$. the bipolar concave integral of $f$ respect to bi-capacity $\mu_{b}$ is defined by

$$
(B L) \int f d v_{b}=\bigvee\left\{\sum_{i=1}^{n} \propto_{i} v_{b}\left(A_{i}, B_{i}\right): \sum_{i=1}^{n} \propto_{i} I_{\left(A_{i}, B_{i}\right)} \leq f, \propto_{i} \geq 0, \quad n \in \mathbb{N}\right\}
$$




\section{Practical Example}

Let us try to illustrate the generalized concave integral (bipolar concave integral) by a case of evaluation of students from an example very well known in the specialized literature [13].

In the admission process, the Director of the College seeks to make a sound decision regarding the order of the students in the College who apply for Postgraduate Studies in Economics where certain prerequisites are required. There are 3 criteria (Mathematics (M), Statistics (S), and Languages (L)) serve as a basis for the preselection of the candidates. We suppose that the students are evaluated on the same bipolar scale from -10 to 10 , with a neutral value of 0 .

Let us consider five students (A, B, C, D, and E) having the evaluations presented in Table 1, and assume that the bi-capacity values as shown in Table 2 . In the next section, we show that the generalized concave integral can model and solve this problem.

Table 1: Degrees of students

\begin{tabular}{|c|c|c|c|}
\hline Subjects & Mathematic (M) & Statistic (S) & Language (L) \\
\hline Students & 4 & 6 & -3 \\
\hline Student A & 3 & 4 & -2 \\
\hline Student B & -1 & 6 & -3 \\
\hline Student C & -1 & 5 & -2 \\
\hline Student D & 5 & 3 & -1 \\
\hline
\end{tabular}

Table 2: Bi-capacity values

\begin{tabular}{|c|c|c|c|c|c|c|c|c|}
\hline$v_{b}(A, B)$ & $\varnothing$ & $\{\mathrm{M}\}$ & $\{\mathrm{S}\}$ & $\{\mathrm{L}\}$ & $\{\mathrm{M}, \mathrm{S}\}$ & $\{\mathrm{M}, \mathrm{L}\}$ & $\{\mathrm{S}, \mathrm{L}\}$ & $\{\mathrm{M}, \mathrm{S}, \mathrm{L}\}$ \\
\hline$\varnothing$ & 0 & -2 & -2 & -2 & -6 & -4 & -6 & -10 \\
\hline$\{\mathrm{M}\}$ & 2 & - & -2 & -2 & - & - & -6 & - \\
\hline$\{\mathrm{S}\}$ & 2 & -3 & - & -3 & - & -6 & - & - \\
\hline
\end{tabular}




\begin{tabular}{|c|c|c|c|c|c|c|c|c|}
\hline$\{\mathrm{L}\}$ & 2 & -2 & -2 & - & -4 & - & - & - \\
\hline$\{\mathrm{M}, \mathrm{S}\}$ & 6 & - & - & 3 & - & - & - & - \\
\hline$\{\mathrm{M}, \mathrm{L}\}$ & 4 & - & 2 & - & - & - & - & - \\
\hline$\{\mathrm{S}, \mathrm{L}\}$ & 6 & 3 & - & - & - & - & - & - \\
\hline$\{\mathrm{M}, \mathrm{S}, \mathrm{L}\}$ & 10 & - & - & - & - & - & - & - \\
\hline
\end{tabular}

\section{Results and discussion}

The decompositions of the considered functions (students: A, B, C, D, and E) are shown in the following tables (Table 3, 4, 5, 6, 7):

Table 3: Decomposition of student A

\begin{tabular}{|c|c|c|}
\hline 1. & $4 v_{b}(\{M\}, \varnothing)+6 v_{b}(\{S\}, \emptyset)+3 v_{b}(\varnothing,\{L\})$ & $4(2)+6(2)+3(-2)=14$ \\
\hline 2. & $4 v_{b}(\{M, S\}, \emptyset)+2 v_{b}(\{S\}, \emptyset)+3 v_{b}(\varnothing,\{L\})$ & $4(6)+2(2)+3(-2)=22$ \\
\hline 3. & $3 v_{b}(\{M, S\},\{L\})+v_{b}(\{M, S\}, \varnothing)+2 v_{b}(\{S\}, \emptyset)$ & $3(3)+(6)+2(2)=19$ \\
\hline 4. & $3 v_{b}(\{M, S\},\{L\})+v_{b}(\{M\}, \varnothing)+3 v_{b}(\{S\}, \varnothing)$ & $3(3)+(2)+3(2)=17$ \\
\hline 5. & $4 v_{b}(\{M, S\}, \emptyset)+2 v_{b}(\{S\},\{L\})+v_{b}(\varnothing,\{L\})$ & $4(6)+2(-3)+(-2)=16$ \\
\hline 6. & $3 v_{b}(\{M, S\}, \emptyset)+3 v_{b}(\{S\},\{L\})+v_{b}(\{M\}, \emptyset)$ & $3(6)+3(-3)+(2)=11$ \\
\hline 7. & $3 v_{b}(\{S\},\{L\})+4 v_{b}(\{M\}, \varnothing)+3 v_{b}(\{S\}, \varnothing)$ & $3(-3)+4(2)+3(2)=5$ \\
\hline 8. & $3 v_{b}(\{M\},\{L\})+v_{b}(\{M, S\}, \emptyset)+5 v_{b}(\{S\}, \varnothing)$ & $3(-2)+(6)+5(2)=10$ \\
\hline 9. & $3 v_{b}(\{M\},\{L\})+v_{b}(\{M\}, \emptyset)+6 v_{b}(\{S\}, \emptyset)$ & $3(-2)+(2)+6(2)=8$ \\
\hline
\end{tabular}

Table 4: Decomposition of student B

\begin{tabular}{|r|c|l|}
\hline 1. & $3 v_{b}(\{M\}, \emptyset)+4 v_{b}(\{S\}, \emptyset)+2 v_{b}(\varnothing,\{L\})$ & $3(2)+4(2)+2(-2)=10$ \\
\hline 2. & $3 v_{b}(\{M, S\}, \emptyset)+v_{b}(\{S\}, \emptyset)+2 v_{b}(\varnothing,\{L\})$ & $3(6)+(2)+2(-2)=16$ \\
\hline 3. & $2 v_{b}(\{M, S\},\{L\})+v_{b}(\{M, S\}, \emptyset)+v_{b}(\{S\}, \emptyset)$ & $2(3)+(6)+(2)=14$ \\
\hline 4. & $2 v_{b}(\{M, S\},\{L\})+v_{b}(\{M\}, \emptyset)+2 \mu_{b}(\{S\}, \emptyset)$ & $2(3)+(2)+2(2)=12$ \\
\hline 5. & $3 v_{b}(\{M, S\}, \emptyset)+v_{b}(\{S\},\{L\})+v_{b}(\varnothing,\{L\})$ & $3(6)+(-3)+(-2)=13$ \\
\hline
\end{tabular}




\begin{tabular}{|r|c|l|}
\hline 6. & $2 v_{b}(\{M, S\}, \emptyset)+2 v_{b}(\{S\},\{L\})+v_{b}(\{M\}, \emptyset)$ & $2(6)+2(-3)+(2)=8$ \\
\hline 7. & $2 v_{b}(\{S\},\{L\})+3 v_{b}(\{M\}, \emptyset)+2 v_{b}(\{S\}, \emptyset)$ & $2(-3)+3(2)+2(2)=4$ \\
\hline 8. & $2 v_{b}(\{M\},\{L\})+v_{b}(\{M, S\}, \emptyset)+3 v_{b}(\{S\}, \emptyset)$ & $2(-2)+(6)+3(2)=8$ \\
\hline 9. & $2 v_{b}(\{M\},\{L\})+v_{b}(\{M\}, \varnothing)+4 v_{b}(\{S\}, \emptyset)$ & $2(-2)+(2)+4(2)=6$ \\
\hline
\end{tabular}

Table 5: Decomposition of student $\mathbf{C}$

\begin{tabular}{|r|c|l|}
\hline 1. & $v_{b}(\varnothing,\{M\})+6 v_{b}(\{S\}, \emptyset)+3 v_{b}(\varnothing,\{L\})$ & $(-2)+6(2)+3(-2)=4$ \\
\hline 2. & $v_{b}(\{S\},\{M, L\})+2 v_{b}(\{S\},\{L\})+3 v_{b}(\{S\}, \emptyset)$ & $(-6)+2(-1)+3(2)=-2$ \\
\hline 3. & $v_{b}(\{S\},\{M, L\})+5 v_{b}(\{S\}, \emptyset)+2 v_{b}(\varnothing,\{L\})$ & $(-6)+5(2)+2(-2)=0$ \\
\hline 4. & $3 v_{b}(\{S\},\{L\})+3 v_{b}(\{S\}, \emptyset)+v_{b}(\varnothing,\{M\})$ & $3(-3)+3(2)+(-2)=-5$ \\
\hline 5. & $v_{b}(\varnothing,\{M, L\})+2 v_{b}(\{S\},\{L\})+4 v_{b}(\{S\}, \emptyset)$ & $(-4)+2(-3)+4(2)=-2$ \\
\hline 6. & $v_{b}(\varnothing,\{M, L\})+6 v_{b}(\{S\}, \emptyset)+2 v_{b}(\varnothing,\{L\})$ & $(-4)+6(2)+2(-2)=4$ \\
\hline 7. & $v_{b}(\{S\},\{M\})+3 v_{b}(\{S\},\{L\})+2 v_{b}(\{S\}, \emptyset)$ & $(-3)+3(-3)+2(2)=-8$ \\
\hline 8. & $v_{b}(\{S\},\{M\})+5 v_{b}(\{S\}, \emptyset)+3 v_{b}(\varnothing,\{L\})$ & $(-3)+5(2)+3(-2)=1$ \\
\hline
\end{tabular}

Table 6: Decomposition of student $D$

\begin{tabular}{|r|c|l|}
\hline 1. & $v_{b}(\varnothing,\{M\})+5 v_{b}(\{S\}, \emptyset)+2 v_{b}(\varnothing,\{L\})$ & $(-2)+5(2)+2(-2)=4$ \\
\hline 2. & $v_{b}(\{S\},\{M, L\})+v_{b}(\{S\},\{L\})+3 v_{b}(\{S\}, \emptyset)$ & $(-6)+(-3)+3(2)=-3$ \\
\hline 3. & $v_{b}(\{S\},\{M, L\})+4 v_{b}(\{S\}, \emptyset)+v_{b}(\varnothing,\{L\})$ & $(-6)+4(2)+(-2)=0$ \\
\hline 4. & $2 v_{b}(\{S\},\{L\})+3 v_{b}(\{S\}, \emptyset)+v_{b}(\varnothing,\{M\})$ & $2(-3)+3(2)+(-2)=-2$ \\
\hline 5. & $v_{b}(\varnothing,\{M, L\})+v_{b}(\{S\},\{L\})+4 v_{b}(\{S\}, \emptyset)$ & $(-4)+(-3)+4(2)=1$ \\
\hline 6. & $v_{b}(\varnothing,\{M, L\})+5 v_{b}(\{S\}, \emptyset)+v_{b}(\varnothing,\{L\})$ & $(-4)+5(2)+(-2)=4$ \\
\hline 7. & $v_{b}(\{S\},\{M\})+2 v_{b}(\{S\},\{L\})+2 v_{b}(\{S\}, \emptyset)$ & $(-3)+2(-3)+2(2)=-5$ \\
\hline 8. & $v_{b}(\{S\},\{M\})+4 v_{b}(\{S\}, \emptyset)+2 v_{b}(\varnothing,\{L\})$ & $(-3)+4(2)+2(-2)=1$ \\
\hline
\end{tabular}


Table 7: Decomposition of student $\mathrm{E}$

\begin{tabular}{|r|c|l|}
\hline 1. & $5 v_{b}(\{\mathrm{M}\}, \emptyset)+3 v_{b}(\{\mathrm{~S}\}, \emptyset)+v_{b}(\varnothing,\{\mathrm{L}\})$ & $5(2)+3(2)+1(-2)=14$ \\
\hline 2. & $v_{b}(\{\mathrm{M}, \mathrm{S}\},\{\mathrm{L}\})+2 v_{b}(\{\mathrm{MS}\}, \emptyset)+2 v_{b}(\{\mathrm{M}\}, \emptyset)$ & $(3)+2(6)+2(2)=19$ \\
\hline 3. & $v_{b}(\{\mathrm{M}, \mathrm{S}\},\{\mathrm{L}\})+2 v_{b}(\{\mathrm{~S}\}, \emptyset)+4 v_{b}(\{\mathrm{M}\}, \emptyset)$ & $(3)+2(2)+4(2)=15$ \\
\hline 4. & $v_{b}(\{\mathrm{M}\},\{\mathrm{L}\})+3 v_{b}(\{\mathrm{M}, \mathrm{S}\}, \emptyset)+v_{b}(\{\mathrm{M}\}, \emptyset)$ & $1(-2)+3(6)+1(2)=18$ \\
\hline 5. & $v_{b}(\{\mathrm{M}\},\{\mathrm{L}\})+4 v_{b}(\{\mathrm{M}\}, \emptyset)+3 v_{b}(\{\mathrm{~S}\}, \emptyset)$ & $1(-2)+4(2)+3(2)=12$ \\
\hline 6. & $3 v_{b}(\{\mathrm{M}, \mathrm{S}\}, \emptyset)+2 v_{b}(\{\mathrm{M}\}, \emptyset)+v_{b}(\varnothing,\{\mathrm{L}\})$ & $3(6)+2(2)+1(-2)=20$ \\
\hline 7. & $v_{b}+5 v_{b}(\{\mathrm{M}\}, \emptyset)+2 v_{b}(\{\mathrm{~S}\}, \emptyset)$ & $1(-3)+5(2)+2(2)=11$ \\
\hline 8. & $v_{b}(\{\mathrm{~S}\},\{\mathrm{L}\})+2 v_{b}(\{\mathrm{M}, \mathrm{S}\}, \emptyset)+3 v_{b}(\{\mathrm{M}\}, \emptyset)$ & $1(-3)+2(6)+3(2)=15$ \\
\hline
\end{tabular}

When we need the best candidates without restriction, the bipolar concave integral is suitable for finding the solution based on an optimal decomposition of the functions (students: A, B, C, D, and E). Therefore, we will use it to find the solution.

$$
\begin{aligned}
& (B L) \int f d v_{b}=\bigvee\left\{\sum_{i=1}^{n} \propto_{i} v_{b}\left(A_{i}, B_{i}\right): \sum_{i=1}^{n} \propto_{i} I_{\left(A_{i}, B_{i}\right)} \leq f, \propto_{i} \geq 0, n \in \mathbb{N}\right\} . \\
& (B L) \int A d v_{b}=4 v_{b}(\{M, S\}, \emptyset)+2 v_{b}(\{S\}, \emptyset)+3 v_{b}(\varnothing,\{L\})=22 \\
& (B L) \int B d v_{b}=3 v_{b}(\{M, S\}, \emptyset)+v_{b}(\{S\}, \emptyset)+2 v_{b}(\varnothing,\{L\})=16 \\
& (B L) \int C d v_{b}=v_{b}(\varnothing,\{M\})+6 v_{b}(\{S\}, \emptyset)+3 v_{b}(\varnothing,\{L\})=4 \\
& (B L) \int D d v_{b}=v_{b}(\varnothing,\{M\})+5 v_{b}(\{S\}, \varnothing)+2 v_{b}(\varnothing,\{L\})=4 \\
& (B L) \int E d v_{b}=3 v_{b}(\{\mathrm{M}, \mathrm{S}\}, \emptyset)+2 v_{b}(\{\mathrm{M}\}, \varnothing)+v_{b}(\varnothing,\{\mathrm{L}\})=20
\end{aligned}
$$

Table 8: Results 


\begin{tabular}{|c|c|c|c|c|}
\hline Subjects & Mathematic (M) & Statistic (S) & Language (L) & $\begin{array}{c}\text { Evaluation by } \\
\text { bipolar concave Integral }\end{array}$ \\
\hline Students & 4 & 6 & -3 & 22 \\
\hline Student B & 3 & 4 & -2 & 46 \\
\hline Student C & -1 & 6 & -3 & 4 \\
\hline Student B & -1 & 5 & -2 & 20 \\
\hline Student E & 5 & 3 & -1 & 4 \\
\hline
\end{tabular}

From Table 8 , the highest value of bipolar concave integral values is 22 . Therefore, we conclude that student $\mathrm{A}$ is the best of the students and should be admitted first for graduate studies in economics.

\section{Conclusions}

This study introduced bipolar concave integral in terms of the decomposition of the integral function and illustrated our framework by a practical example. In this integration, the random variable is analyzed as a combination of indicators, where each allowed decomposition has a value determined by the bi-capacity.

We believe that concave's integral concept will allow for new theoretical developments, wherein bipolarity is included in choices, both in multi-criteria decision-making and decisions under risk and uncertainty.

The proposed results are consistent as generalizations of the concave integral for capacities. Therefore, we trust that other integrals [12] [18-20] may be generalized in a similar manner. Also, one of the topics of our subsequent researches will be a discussion and examination of the properties and relationship of the bipolar concave integral with other integrals. Because of the high potential computational complexity, efficient algorithms are needed to compute bipolar concave integral. Therefore, some algorithms used in applications can be developed [21-23]).

\section{Acknowledgements}

The authors wish to thank the anonymous reviewers for their careful reading and valuable suggestions, which helped to improve the original version of this contribution.

\section{Conflict of Interest}

The authors declare that they have no known competing financial interests or personal relationships that could have appeared to influence the work reported in this paper.

\section{References}

[1] G. Choquet, “Theory of capacities”,Ann. Inst. Fourier, Vol. 5, pp. 131-295, 1953.

[2] M. Grabisch, T. Murofushi,, Sugeno, M., "Fuzzy Measures and Integrals. Theory and Applications", Physica Verlag, Berlin Heidelberg, 2000.

[3] N. Shilkret, "Maxitive measure and integration", Indag. Math., Vol.33, PP. 109-116, 1971.

[4] R. I. Sabri, M. N. Mohammedali, and J. Abbas, "An application of non-additive measures and corresponding integrals in tourism management", Baghdad Science Journal, vol.17, No.1, pp.172$177,2020$. 
[5] M. Hesham and J. Abbas, "Multi-criteria decision making on the optimal drug for rheumatoid arthritis disease", Iraqi Journal of Science, vol.62, no.5, 2021.

[6] J. Abbas, "Shilkret Integral Based on Binary-Element Sets and its Application in The Area of Synthetic Evaluation", Engineering \& Technology Journal, Vol.33, Part (B), No.3, 2015.

[7] J. Abbas, "Logical twofold integral”, Engineering \& Technology Journal, Vol. 28, No. 3; 2010.

[8] A. Mendez, P. Gader, J. Keller, K. Chamberlin, "Minimum classification error training for Choquet integrals with applications to landmine detection", IEEE Transactions on Fuzzy Systems, Vol 16, No.1, pp. 225-238, 2008.

[9] J. Abbas, H. Ali, "Evaluation of Baghdad water quality using Fuzzy logic", Iraqi Journal of Science, Vol. 58, No.2C, pp: 1128-1135, 2017.

[10] J. Abbas, M. Israa, "A Generalized Integral of Shilkret and Choquet Integrals", Iraqi Journal of Science, Vol. 57, No.3A, pp.1813-1818, 2016.

[11] M. Grabisch, C. Labreuche, "Bi-capacities I: Definition, Mobius transform and interaction", Fuzzy Sets and Systems, Vol. 151, pp. 211-236, 2005.

[12] M. Grabisch and C. Labreuche, "Bi-capacities II: The Choquet integral", Fuzzy Sets and Systems, Vol. 151, pp. 237-259, 2005.

[13] S. Greco, F. Rindone, "Bipolar fuzzy integrals", Fuzzy Sets and Systems, Vol.220, PP. 21- 33, 2013.

[14] J. Abbas, "The Balancing Bipolar Choquet Integrals", International Journal Of Innovative Computing, Information And Control, Vol.17, No.3, PP. 949-957, 2021.

[15] E. Lehrer, “A new integral for capacities”, Econ. Theory, Vol. 39, PP. 157-176, 2009.

[16] J. M. Bilbao, Fernandez, J.R., Jimenez Losada, A. and Lebron, E., "Bicooperative

Games", First World Congress of the Game Theory Society, Games 2000, July 24-28, Spain, 2000.

[17] J. Abbas, "The Banzhaf interaction index for bi-cooperative games", International Journal of General Systems, Vol.50, No 5, PP. 486-500, 2021.

[18] J. Abbas, "Bipolar Choquet integral of fuzzy events", IEEE SSCI conference on MCDM, Florida, USA, pp.116-123, 2014.

[19] J. Abbas, "The Bipolar Choquet Integrals Based On Ternary-Element Sets", Journal Of Artificial Intelligence And Soft Computing Research, Vol. 6, No. 1, pp. 13-21, 2016.

[20] J. Abbas, "The 2-Additive Choquet Integral Of Bi-Capacities”, In: Rutkowski L., Scherer R., Korytkowski M., Pedrycz W., Tadeusiewicz R., Zurada J. (eds) Artificial Intelligence and Soft Computing, ICAISC 2019, Lecture Notes in Computer Science, Vol. 11508, Springer, 2019.

[21] F. A. Sadiq, R. Jalal, J. Abbas, "A computational mechanism for making admission decisions in the centralized admission system”, ICCEPS-2021, AIP Conference Proceedings, accepted.

[22] A. Naeem and J. Abbas, "A Computational Model for Multi-Criteria Decision Making in Traffic Jam Problem", Journal of Automation, Mobile Robotics and Intelligent Systems, accepted.

[23] B. Kamal, Nadia Al-Saidi, "Extended Chaotic Nonlinear Programming Technique Constructing with Genetic Algorithms", Journal of Applied Sciences and Nanotechnology, Vol.1, No.1, 15-22, 2021. 